Fortschr. Röntgenstr. 141, 3 (1984) 306-313

(c) Georg Thieme Verlag Sturtgart - New York

\section{Das metastasierende differenzierte Schilddrüsenkarzinom} Diagnostische Treffsicherheit des Thyreoglobulin-RIA im Vergleich zur ${ }^{131} \mathrm{~J}$-Ganzkörper-
szintigraphie

Von Chr. Reiners, J. Reimann, R. Schäffer *, K. Baum, W. Becker, Chr. Eilles, W. Gerhards, F. Schick, W. Spiegel, W. Wiedemann und W. Börner

\section{Abbildungen}

Abtcilung für Nuklearmedizin (Leiter: Prof. Dr. W. Börner) und Pathologisches Institut" (Vorstand: Prof. Dr. Dr. h.c. H.-W. Altmann) der Universität Würzburg

In einer retrospektiven Analyse an Patienten mit differenziertem Schilddrüsenkarzinom werden die diagnostischen Treffsicherheiten der ${ }^{131} \mathrm{~J}$-Ganzkörperszintigraphie und der radioimmunologischen Bestimmung des Tumormarkers Thyreoglobulin (hTg) unter besonderer Berücksichtigung der Erfassung von Spätmetastasen verglichen. Bei 83 von 311 Patienten lag eine Metastasierung vor. Dabei handelte es sich in zwei Drittel der Fälle um Primärmetastasen, im restlichen Drittel der Fälle traten Spätmetastasen mit einer durchschnittlichen Latenzzeit von 3,3 Jahren auf. Während rund $70 \%$ der Primärmetastasen durch die ${ }^{131} \mathrm{~J}$-Szintigraphie erfaßt wurden, betrug dieser Prozentsatz bei den Spätmetastasen nur rund $40 \%$. Die diagnostische Treffsicherheit des hTg-RIA war hier mit einer Sensitivität von rund $90 \%$ sowohl für Primär- als auch für Spätmetastasen deutlich überlegen. Allerdings war das hTg bei 4 von 49 Fällen nur in iatrogener Hypothyreose meßbar. Basierend auf diesen Ergebnissen und einer Literaturanalyse wird ein Programm für die Verlaufskontrolle des differenzierten Schilddrüsenkarzinoms vorgestellt. Der hTg-RIA findet dabei nach vollständiger Ablation allen Schilddrüsengewebes in der Spätphase der Nachsorge als Alternative zur ${ }^{131} \mathrm{~J}$-Szintigraphie Verwendung.

\begin{abstract}
Metastasizing differentiated thyroid carcinoma: diagnostic sensitivity of thyroglobulin-RIA in comparison to whole-body scintigraphy with ${ }^{131}$ I
\end{abstract}

In a retrospective analysis in patients with differentiated thyroid carcinoma, the diagnostic validities of ${ }^{131}$ I whole-body scans and radioimmunologic determinations of thyroglobulin ( $\mathrm{h} \mathrm{Tg}$ ) are compared with special regard to late merastases. Metastases were found in 83 out of 311 patients with differentiated thyroid carcinoma. In two thirds of the cases, these were primary metastases while in the remaining third of the cases, metastases developed in later follow-up with a mean time of latency of 3.3 years. While about $70 \%$ of the early metastases could bei detected by ${ }^{131}$ I scintigraphy, this percentage amounted to only $40 \%$ in late metastases. With a diagnostic sensitivity of $90 \%$, hTg-RIA was clearly superior in the detection of early as well as of late metastases. $\mathrm{hTg}$ was measurable, however, only in iatrogenous hypothyroidism in 4 out of 49 cases. Based on these results and an analysis of the literature, a program for follow-up of differentiated thyroid carcinoma is proposed. The $\mathrm{hTg}$-RIA is thereby used as an alternative to ${ }^{131}$ I scintigraphy in the late phase of follow-up after complete ablation of any thyroid tissue.
Die Prognose des differenzierten Schilddrüsenkarzinoms ist insbesondere bei Verwendung standardisierter Therapie- und Nachsorgeprogramme $(6,7,43)-\mathrm{im}$ allgemeinen als sehr gut zu betrachten (Tab. 1). So liegen die 5-Jahres- und 10-JahresÜberlebensraten des papillären und des hoch differenzierten follikulären Karzinoms bei rund $90 \%$. Beim onkozytären Karzinom - früher als Hürthle-Zell-Karzinom bezeichnet -, das wir wegen seines besonderen biologischen Verhaltens getrennt betrachten (u. a. fehlende Radiojodspeicherung (47)), finden sich nur wenig ungünstigere Überlebensraten. Nur die gering differenzierte Form des follikulären Karzinoms weist eine merklich schlechtere Prognose auf (vgl. Tab. 1).

Wenn auch die Inzidenz des Schilddrüsenkarzinoms relativ gering ist (rund 40 neue Fälle pro Jahr in unserem Untersuchungsgut), so nimmt doch die Zahl der in die Nachsorge zu übernehmenden Patienten aufgrund der guten Prognose beständig zu. Ein diagnostisches Nachsorgeprogramm verlangt gerade beim differenzierten Schilddrüsenkarzinom nach einer auf der einen Seite möglichst sensitiven, auf der anderen Seite aber wenig aufwendigen und den Patienten nicht belästigenden Methode zur Erfassung von Tumorrezidiven oder Metastasen. Wir sind aus diesem Grunde der bereits auch von anderen Arbeitsgruppen diskutierten Frage nachgegangen, ob die radioimmunologische Bestimmung des Thyreoglobulins im Serum die bisher im Rahmen der Nachsorge allgemein üblichen ${ }^{131} \mathrm{~J}$ Ganzkörperszintigramme ersetzen kann. Dabei wurde die diagnostische Treffsicherheit der beiden Methoden zur Erfassung von Spätmetastasierungen der verschiedenen histologischen Typen des differenzierten Schilddrüsenkarzinoms besonders geprït.

\section{Patienten und Methoden}

Seit mehr als 10 Jahren erfolgt die Behandlung von Patienten mit differenziertem Schilddrüsenkarzinom an den Würzburger Universitätskliniken nach einem einheitlichen Therapiekonzept, das aus einer

Tab. 1 Überlebenswahrscheinlichkeiten (life-table-Methode) für das differenzierte Schilddrüsenkarzinom (39).

\begin{tabular}{lll}
\hline histologischer Typ & \multicolumn{2}{c}{$\begin{array}{l}\text { Uberlebenswahrscheinlichkeiten } \\
5 \text { Jahre }\end{array}$} \\
& $88 \%$ & $88 \%$ \\
\hline papillär & $90 \%$ & $90 \%$ \\
follikul. hoch diff. & $63 \%$ & $55 \%$ \\
follikul, wenig diff. & $87 \%$ & $67 \%$ \\
onkozytär & \\
\hline
\end{tabular}


totalen Thyreoidektomie und der anschließenden Elimination allen speichernden Schilddrüsengewebes durch die Radiojodbehandlung besteht. An dieser Stelle ist zu erwähnen, daß die Radiojodtherapie aufgrund der in Würzburg zur Zeit noch fehlenden baulichen Voraussetzungen in der Universitäts-Strahlenklinik Heidelberg (Direktor: Professor Dr. K. zum Winkel) durchgeführt wurde; den Heidelberger Kollegen ist für die stets entgegenkommende Zusammenarbeit zu danken. Ausnahmen von dem radikalen Therapiekonzept wurden nur beim okkulten papillären Mikrokarzinom jüngerer Patienten sowie beim nicht radiojodspeichernden onkozytären Karzinom gemacht.

Tab. 2 gibt eine Übersicht über das Untersuchungsgut der vorliegenden Studie. Insgesamt wurden die Verläufe von 311 Patienten ausgewertet. Die histologische Klassifikation der Schilddrüsentumoren erfolgte durch einen der Mitautoren ( $R$. Schäffer). Auch bei Tumoren, von denen zunächst ein auswärtiger Histologiebefund vorlag, wurde in den meisten Fällen eine Nachbegutachtung durch den Pathologen unserer Arbeitsgruppe vorgenommen, so daß von einer einheitlichen Klassifikation der Schilddrüsenmalignome ausgegangen werden kann. Abweichend von der derzeit allgemein angewendeten WHO-Klassifikation der Schilddrüsentumoren betrachten wir das onkozytäre Karzinom, das in dieser Klassifikation unter den papillären bzw. follikulären Karzinomen subsummiert ist, aus den bereits angegebenen Gründen gesondert (47).

Die Therapie erfolgte nach folgendem Schema $(6,7)$ : Nach der totalen Thyreoidektomie wurde zunächst eine Radiojodtherpie mit $70 \mathrm{mCi}$ ${ }^{131} \mathrm{~J}$ durchgeführt. Beim Vorhandensein von Restspeicherungen im Schilddrüsenbett oder speichernden Metastasen folgten im Abstand von 3-4 Monaten weitere Radiojodbehandlungen mit $100 \mathrm{mCi}$. Dieses Verfahren wurde so lange fortgesetzt, bis im Posttherapieszintigramm keine Speicherung mehr im Schilddrüsenbett oder über ehedem speichernden Metastasen nachzuweisen war. Zwischen den Radiojodbehandlungen und nach Abschluß der Radiojodtherapie erfolgte eine suppressive Hormontherapie mit individuell nach dem TRH-Test eingestellten Dosen von L-Thyroxin.

Auch für die Nachsorge wurde ein einheitliches Schema verwendet ( 7 , 43). Dieses besteht im wesentlichen aus:

1. klinischer Untersuchung, Schilddrüsen-in-vitro-Tests sowie weiteren Laboruntersuchungen wie BSG, alkalischer Phosphatase, Gamma-GT etc. in etwa halbjährlichen Abständen. Seit 1979 werden diese Untersuchungen durch die regelmäßige Bestimmung des Serum-Thyreoglobulins ( $h \mathrm{Tg}$ ) mit einem kommerziellen Radioimmunassay ergänzt (45). Die Sensitivität dieses Assays kann bei $2,5 \mathrm{ng} / \mathrm{ml}$ angesetzt werden, die Inter-assay-Variabilität ist mit einem Variationskoeffizienten von $9 \%(\bar{x}=35 \mathrm{ng} / \mathrm{ml})$ zufriedenstellend $(42)$.

Zur Erfassung von potentiellen Störungen der hTg-Bestimmung durch endogene Autoantikörper gegen Thyreoglobulin verwenden wir routinemäßig individuelle Wiederfindeversuche; als Normbereich der Wiederfindung betrachten wir Abweichungen von $\pm 30 \%$ um den Sollwert $(42,44)$. Beim metastasierenden Schilddrüsenkarzinom finden wir keine überzufälligen Störungen der Wiederfindung $(42,44)$, die zu Einschränkungen der klinischen Interpretierbarkeit der Ergebnisse des hTg-RIA führen würden.

2. ${ }^{131} J$-Ganzkörperszintigramme 1, 2, 5 und 10 Jahre nach Abschluß der primären Radiojodtherapie. Diese Ganzkörperszintigramme wurden 24 und $72 \mathrm{~h}$ nach $2 \mathrm{mCi}{ }^{131} \mathrm{~J}$ aufgenommen. Zur Erzielung einer optimalen endogenen TSH-Stimulation wurde die Substitutionstherapie mit L-Thyroxin 5 Wochen vor dem Termin der Radiojodszintigraphie für 14 Tage auf $100 \mu \mathrm{g}$ L-Trijodthyronin täglich umgesetzt. Danach folgte ein schilddrüsenhormonfreies Intervall von 3 Wochen.

\section{Ergebnisse}

Häufigkeit, Lokalisation und Zeitpunkt der Metastasierung Abb. 1 und Tab. 3 geben einen Überblick über die Häufigkeit der Metastasierung des differenzierten Schilddrüsenkarzinoms. Dabei wird nach Primärmetastasen (zum Zeitpunkt der Thyreoidektomie bereits bekannt) und Spätmetastasen (mindestens 6 monatiges freies Intervall) unterschieden. Metastasen finden sich in insgesamt 83 von 311 , d.h. rund $30 \%$ der Fälle von differenziertem Schilddrüsenkarzinom. Davon entfallen etwa
$2 / 3$ auf Primär- und $1 / 3$ auf Spätmetastasen. Primärmetastasen sind besonders häufig beim papillären Karzinom. Demgegenüber finden sich Spätmetastasen beim wenig differenzierten follikulären Karzinom etwa doppelt so häufig wie bei den übrigen histologischen Typen.

Abb. 2 zeigt die Verteilung der Latenzzeiten vom Zeitpunkt der histologischen Diagnosestellung bei der Thyreoidektomie bis zum Auftreten von Spätmetastasen. Das Maximum der Häufigkeitsverteilung liegt zwischen 3 und 4 Jahren; in diesem Intervall finder sich auch der Median der Latenzzeiten (3,3 Jahre). Die Verteilung der Latenzzeiten bis zum Auftreten einer Spätmetastasierung ist jedoch ausgesprochen rechtsschief, d. h. daß es in Einzelfällen auch noch mehr als 10 Jahre nach der Primärdiagnose zu einer Spätmetastasierung kommen kann. Eindeutige Unterschiede zwischen den verschiedenen histologischen Typen sind bezüglich des Zeitpunkts der Metastasierung nicht festzustellen.

Tab. 4 schlüsselt die Metastasierung nach der Lokalisation in Abhängigkeit vom histologischen Typ und vom Zeitpunkt des Auftretens der Metastasen auf. Den Zahlen ist zu entnehmen, daß die relativ hohe Häufigkeit von Primärmetastasen beim papillären Karzinom zum einen auf befallene Halslymphknoten, zum anderen auf Lungenmetastasen zurückzuführen ist. Typischerweise treten Halslymphknotenmetastasen und eine diffuse Lungenmetastasierung - insbesondere bei jüngeren Patienten - beim papillären Karzinom relativ häufig gemeinsam auf. Beim hoch differenzierten follikulären Karzinom finden sich im vorliegenden Untersuchungsgut keine Lymphkno-

Tab. 2 Zusammensetzung des Untersuchungsguts nach histologischem Typ des Schilddrüsenkarzinoms, Geschlecht und Lebensalter.

\begin{tabular}{lcccl}
\hline histologischer Typ & \multicolumn{3}{c}{ Häufigkeit } & mittl. Alter \\
& $q$ & $\delta$ & gesamt & $(\bar{x} \pm s$ Jahre $)$ \\
\hline papillär & 90 & 26 & 116 & $42,6 \pm 17,0$ \\
follikul. hoch diff. & 40 & 16 & 56 & $41,6 \pm 17,5$ \\
follikul. wenig diff. & 62 & 19 & 81 & $49,6 \pm 19.2$ \\
onkozytär & 47 & 11 & 58 & $51,1 \pm 15,1$ \\
\hline Gesamtzahl & 239 & 72 & 311 & $46,0 \pm 17,8$ \\
\hline
\end{tabular}

Tab. 3 Absolute und relative Häufigkeiten von Metastasen des differenzierten Schilddrüsenkarzinoms unter Berücksichtigung des Diagnosezeitpunktes.

\begin{tabular}{|c|c|c|c|c|c|}
\hline \multirow[b]{2}{*}{$\begin{array}{l}\text { histologischer } \\
\text { Typ }\end{array}$} & \multirow[b]{2}{*}{$n$} & \multirow[b]{2}{*}{ primär } & \multicolumn{2}{|c|}{ Metastasen } & \multirow[b]{2}{*}{ Summe * } \\
\hline & & & spät & $\begin{array}{l}\text { Latenz } \\
\text { (Monate) }\end{array}$ & \\
\hline papillär & 116 & $31(27 \%)$ & $8(7 \%)$ & $\tilde{x}=45$ & $36(31 \%)$ \\
\hline $\begin{array}{l}\text { follikul. hoch } \\
\text { diff. }\end{array}$ & 56 & $10(18 \%)$ & $4(7 \%)$ & $\bar{x}=75$ & $12(21 \%)$ \\
\hline $\begin{array}{l}\text { follikul. wenig } \\
\text { diff. }\end{array}$ & 81 & $16(20 \%)$ & $12(15 \%)$ & $\bar{x}=37$ & $24(30 \%)$ \\
\hline onkozytär & 58 & $6(10 \%)$ & $5(9 \%)$ & $\tilde{x}=45$ & $11(19 \%)$ \\
\hline Gesamtzahl & 311 & $63(20 \%)$ & $29(9 \%)$ & $\tilde{x}=39$ & $83(27 \%)$ \\
\hline
\end{tabular}

- Summe kleiner als Addition von Primär- und Spätmetastasen, da Patienten mit Spätmetastasen z. T. bereits Primärmetastasen autwiesen. 


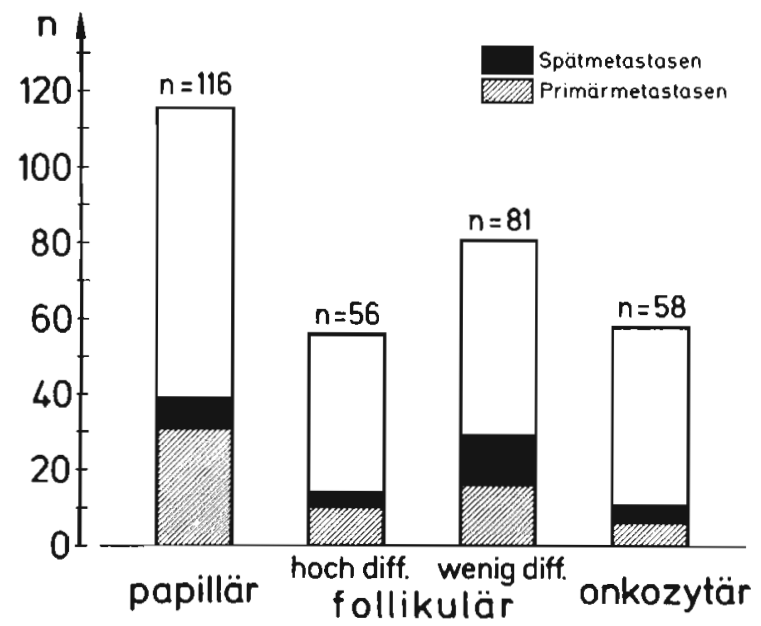

Abb. 1 Aufteilung der Häufigkeit von Primär- und Spätmetastasen auf die verschiedenen histologischen Typen des differenzierten Schilddrüsenkarzinoms.

tenmetastasen oder Lokalrezidive; wenn es zu einer Fernmetastasierung kommt, so ist diese meist primär vorhanden und in der Lunge oder im Skelett lokalisiert. Beim wenig differenzierten follikulären Karzinom fällt zunächst im Vergleich zu den übrigen Tumorformen die insgesamt deutlich höhere Häufigkeit von Spätmetastasen auf. Weiterhin treten hier relativ häufig Lokalrezidive auf; Fernmetastasen befallen meist die Lunge und das Knochensystem. Beim onkozytären Karzinom findet sich keine bevorzugte Lokalisation; Primär- und Spätmetastasen sind etwa gleich häufig.

\section{Jodspeicherverhalten der Metastasen}

Tab. 5 schlüsselt die Metastasierung bei 75 der 83 Patienten mit metastasierendem differenzierten Schilddrüsenkarzinom nach histologischem Typ und Speicherverhalten im Posttherapieszintigramm bzw. im diagnostischen Szintigramm mit $2 \mathrm{mCi}{ }^{131} \mathrm{~J}$ auf. Dabei zeigt sich, daß das Verhältnis von speichernden zu nicht speichernden Metastasen bei der Primärmetastasierung rund 2:1 ist, während sich dieses Verhältnis bei der Spätmetastasierung umkehrt.

Besonders gut speichert erwartungsgemäß das hoch differenzierte follikuläre Karzinom. Etwas ungünstiger schneidet das papilläre Karzinom ab. Die Gruppe der Patienten mit nicht radiojodspeichernden Metastasen des papillären Karzinoms ist dadurch charakterisiert, daß hierin bevorzugt ältere Patienten mit umschriebenen Lungenmetastasen enthalten sind. Das Durchschnittsalter der Patienten mit nicht speichernden Meta-

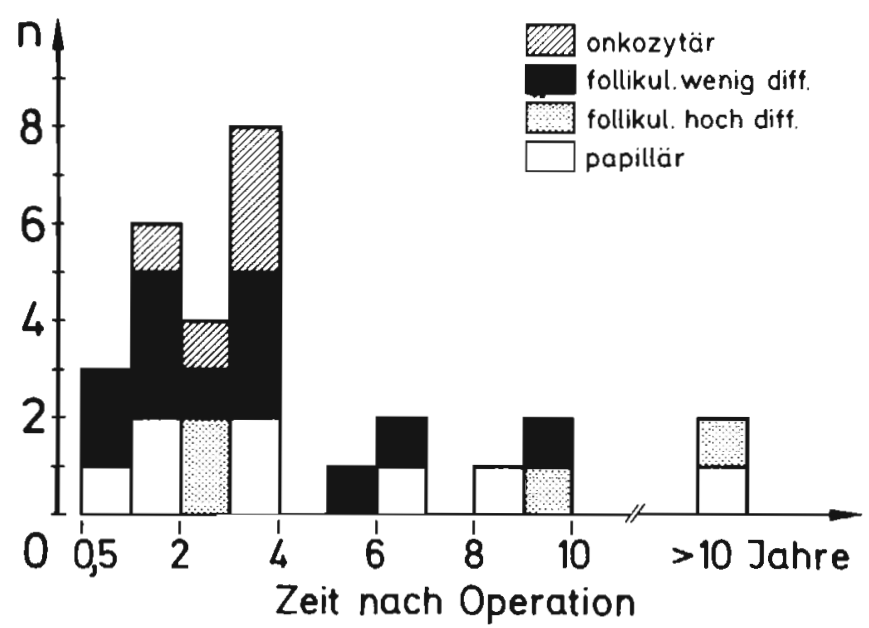

Abb. 2 Häufigkeitsverteilung der Latenzzeiten bis zum Auftreten von Spätmetastasen des differenzierten Schilddrüsenkarzinoms.

stasen des papillären Karzinoms liegt mit 63 Jahren rund 30 Jahre höher als das der Patienten mit speichernden Metastasen dieser Gruppe (32 Jahre). Ähnliche Altersunterschiede finden sich für die übrigen histologischen Typen nicht. Metastasen des wenig differenzierten follikulären Karzinoms speichern in rund $3 / 4$ der Fälle kein Radiojod. Völlig fehlt die Fähigkeit zur Radiojodspeicherung beim onkozytären Karzinom.

Serum-Thyreoglobulin in Abhängigkeit vom Zeitpunkt und Jodspeicherverhalten der Metastasierung

Abb. 3 und Tab. 6 zeigen die verfügbaren Resultate der SerumThyreoglobulin-Bestimmung von 55 der 83 Patienten mit metastasierendem differenzierten Schilddrüsenkarzinom. Als positiv im Sinne einer Metastasierung betrachten wir nach Thyreoidektomie und ablativer Radiojodtherapie ein SerumThyreoglobulin von größer $5 \mathrm{ng} / \mathrm{ml}$. ln insgesamt 49 der 55 Fälle weist die Thyreoglobulin-Bestimmung auf die Metastasierung hin (Tab.6). Es zeigt sich, daß auch nicht radiojodspeichernde Metastasen in der Mehrzahl der Fälle meßbare Thyreoglobulinspiegel aufweisen (offene Kreise in Abb. 3).

Unter endogener TSH-Stimulation nach Absetzen der Thyroxin-Medikation liegt der Mittelwert des Serum-Thyreoglobulins von Patienten mit Primärmetastasen bei $120 \mathrm{ng} / \mathrm{ml}$, während er bei Fällen mit Spätmetastasen $85 \mathrm{ng} / \mathrm{ml}$ beträgt. Die suppressive Thyroxin-Therapie senkt das Serum-Thyreoglobulin bei Primärmetastasen um rund $50 \mathrm{ng} / \mathrm{ml}$, bei Spätmetastasen um rund $30 \mathrm{ng} / \mathrm{ml}$ (vgl. Abb. 3).

Tab. 4 Lokalisation der Metastasen bei 83 Patienten mit differenziertem Schildơrüsenkarzinom.

\begin{tabular}{|c|c|c|c|c|c|c|c|c|c|c|c|}
\hline & $\begin{array}{l}\text { pap } \\
\text { primär }\end{array}$ & 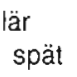 & $\begin{array}{l}\text { follikul. } \\
\text { primär }\end{array}$ & $\begin{array}{l}\text { hoch diff. } \\
\text { spät }\end{array}$ & $\begin{array}{l}\text { follikul. } \\
\text { primär }\end{array}$ & $\begin{array}{l}\text { wenig diff. } \\
\text { spät }\end{array}$ & $\begin{array}{l}\text { onkozy } \\
\text { primär }\end{array}$ & $\begin{array}{l}\text { tär } \\
\text { spät }\end{array}$ & $\begin{array}{l}\text { insgesa } \\
\text { primär }\end{array}$ & $\begin{array}{l}m t \\
\text { spät }\end{array}$ & Summe \\
\hline Halslymphknoten, Lokalrezidive & 25 & 5 & - & - & 3 & 4 & 1 & 2 & 29 & 11 & 40 \\
\hline Lungenmetastasen & 11 & 2 & 4 & 2 & 8 & 6 & 2 & 2 & 25 & 12 & 37 \\
\hline Skelettmetastasen & 2 & - & 6 & 2 & 10 & 5 & 2 & 2 & 20 & 9 & 29 \\
\hline sonstige (Mediastinum, Hirn etc.) & 2 & 2 & 1 & - & 2 & 2 & 1 & 1 & 6 & 5 & 11 \\
\hline Gesamtzahl & 40 & 9 & 11 & 4 & 23 & 17 & 6 & 7 & 80 & 37 & 117 \\
\hline davon Mehrfach-Lokalisationen & 9 & 1 & 1 & - & 7 & 5 & - & 2 & 17 & 8 & 25 \\
\hline
\end{tabular}


Metastasen

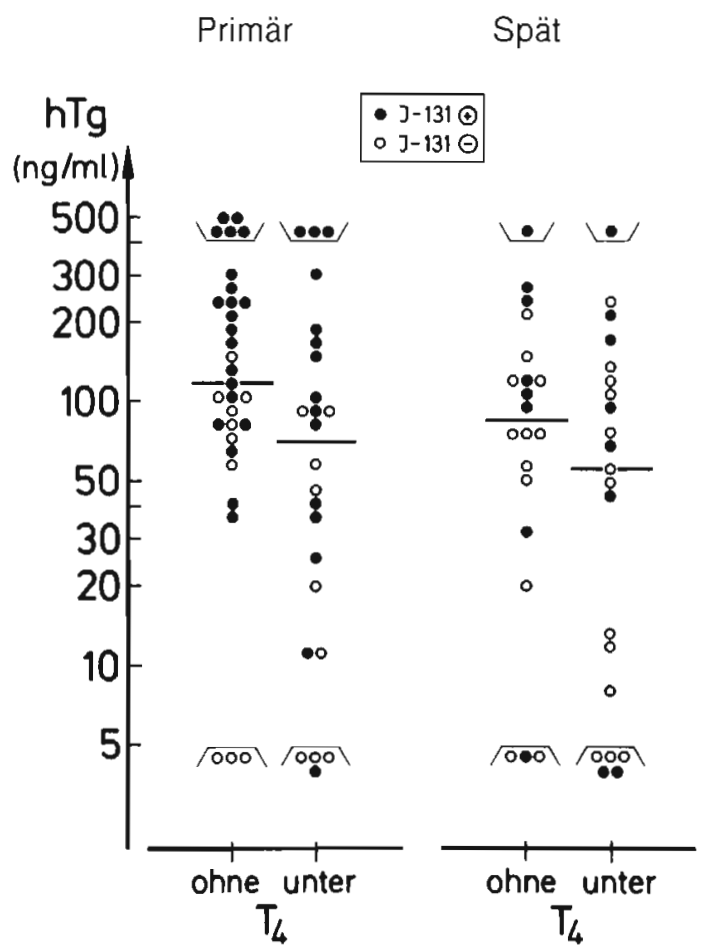

Abb. 3 Verteilung der Serumspiegel des Thyreoglobulins (hTg) bei Patienten mit Primär- und Spätmetastasen des differenzierten Schilddrüsenkarzinoms unter Berücksichtigung des ${ }^{131} \mathrm{~J}$-Speicherverhaltens und des Einflusses der Thyroxin-Therapie (- Median).

Tab. 6 zeigt, daß sich die Zahl der Thyreoglobulin-negativen Metastasen unter Thyroxin-Medikation um 4 erhöht. Eine Abhängigkeit der Treffsicherheit des Thyreoglobulin-RIA vom histologischen Typ des metastasierenden Karzinoms ist der Tab. 6 nicht zu entnehmen.

Abb. 4 faßt die Ergebnisse unserer Studie zur diagnostischen Treffsicherheit der Thyreoglobulin-Bestimmung im Vergleich zur ${ }^{131} \mathrm{~J}$-Ganzkörperszintigraphie zusammen. Es ist eindeutig festzustellen, daß das Resultat des Thyreoglobulin-RIA deutlich häufiger auf die Metastasierung hinweist (in 49 von 55 Fällen) als die Radiojodszintigraphie (in 32 von 55 Fällen). Die Vorteile der Thyreoglobulin-Bestimmung für die Nachsorge zeigen sich vor allem bei der Spätmetastasierung: Hier ist der

Tab. 5 Speicherverhalten der Metastasen im ${ }^{131} \mathrm{~J}$-Ganzkörperszintigramm bei 75 Patienten mit metastasierendem differenzierten Schilddrüsenkarzinom.

\begin{tabular}{lrrrrrr}
\hline histologischer Typ & \multicolumn{3}{l}{$\begin{array}{l}\text { Ergebnisse } \\
\text { Primär- } \\
\text { metastasen }\end{array}$} & $\begin{array}{l}\text { 131 J-Szintigraphie } \\
\text { Spät- } \\
\text { metastasen }\end{array}$ & \multicolumn{2}{l}{ Summe } \\
& $\oplus$ & $\Theta$ & $\oplus$ & $\Theta$ & $\oplus$ & $\Theta$ \\
\hline papillär & 17 & 7 & 4 & 3 & 21 & 10 \\
follikul. hoch diff. & 7 & 2 & 3 & - & 10 & 2 \\
follikul. wenig diff. & 13 & 3 & 3 & 9 & 16 & 12 \\
onkozytär & - & 5 & - & 6 & - & 11 \\
\hline Gesamtzahl & 37 & 17 & 10 & 18 & 47 & 35 \\
\hline
\end{tabular}

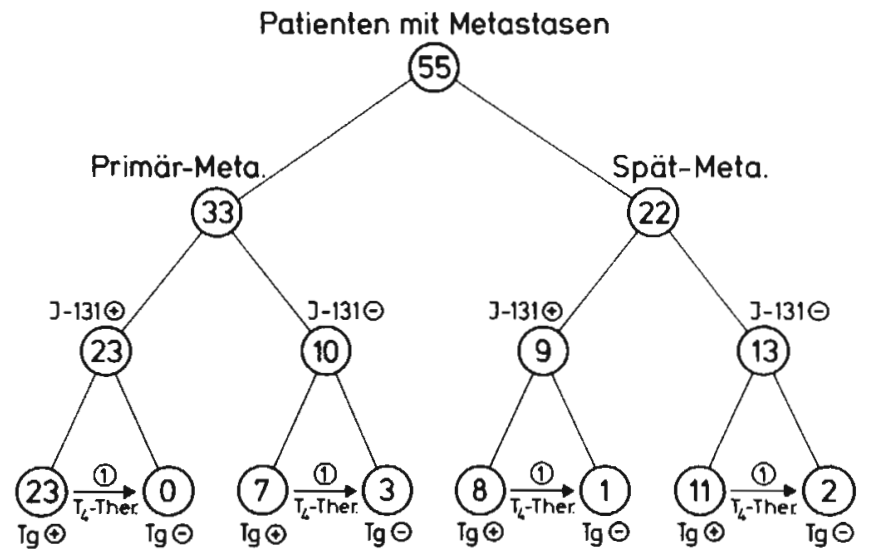

Abb. 4 Aufteilung metastasentypischer Ergebnisse der ${ }^{131} \mathrm{~J}$-Szintigraphie und des Thyreoglobulin(Tg)-RIA bei Patienten mit Primärund Spätmetastasen des differenzierten Schilddrüsenkarzinoms $(\rightarrow$ supprimiertes Tg unter Thyroxin-Therapie).

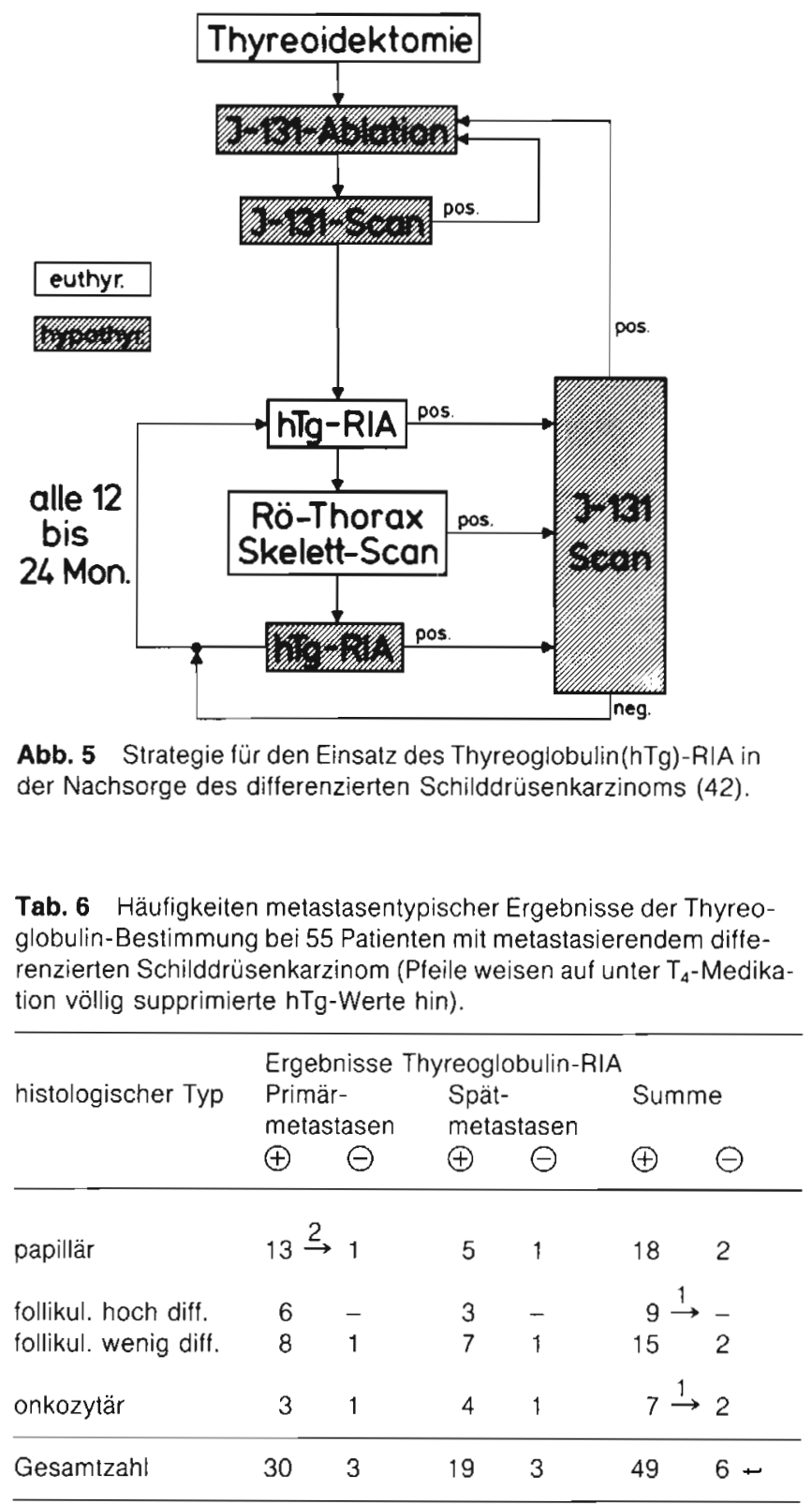


Thyreoglobulin-RIA in 19 von 22 Fällen positiv, während die Radiojodszintigraphie nur in 9 der 22 Fälle die Metastasierung erkennen läßt.

In 4 Fällen wäre eine Metastasierung der ThyreoglobulinBestimmung unter suppressiver Thyroxin-Therapie entgangen. Bei keinem dieser Patienten war jedoch die Metastasierung ausschließlich durch die Radiojodszintigraphie nachweisbar. Sowohl die speichernden als auch die nicht speichernden Metastasen ließen sich auch mit den üblichen zusätzlichen diagnostischen Verfahren, wie klinische Untersuchung, Röntgen-Thoraxaufnahme und Skelettszintigraphie, erkennen.

\section{Diskussion}

\section{Häufigkeit, Zeitpunkt und Jodspeicherverhalten der Metasta-} sierung

Nach den eigenen Untersuchungen finden sich in rund $30 \%$ der Fälle von differenziertem Schilddrüsenkarzinom Metastasen. Bei den Angaben zur Metastasenhäufigkeit in der Literatur fällt die von amerikanischen Autoren beschriebene hohe Inzidenz von Lymphknotenmetastasen in über $50 \%$ der Fälle von papillärem Karzinom auf $(31,32)$. Deutsche Arbeitsgruppen machen diese Beobachtung nicht; im eigenen Untersuchungsgut beträgt diese Häufigkeit $26 \%$. Insgesamt werden beim differenzierten Schilddrüsenkarzinom in $20(24)$ bis $50 \%(14$, 20, 51) der Fälle Lymphknoten- und/oder Fernmetastasen beschrieben.

Im eigenen Untersuchungsgut verhalten sich die Inzidenzen von Primär-zu Spätmetastasen wie $2: 1$; insgesamt kommt es in rund $10 \%$ der Fälle von differenziertem Schilddrüsenkarzinom zu einer Metastasierung nach einem krankheitsfreien Intervall. Spätmetastasen scheinen beim wenig differenzierten follikulären Karzinom etwas häufiger aufzutreten als bei den übrigen differenzierten Schilddrüsenkarzinomen. Die durchschnittliche Latenzzeit beträgt 3,3 Jahre. Bezüglich der Häufigkeit (11-16\%) und der mittleren Latenzzeit (4-4,5 Jahre) stimmen die Angaben amerikanischer $(32,59)$ und französischer Arbeitsgruppen (18) recht gut mit den eigenen Beobachtungen überein. Beierwaltes und Mitarb. (4) beschreiben sogar mit 7,4 Jahren eine deutlich höhere mittlere Latenzzeit. Der Arbeit von Hüfner und Mitarb. (23) ist demgegenüber ein Intervall von rund 3 Jahren bis zum Auftreten der Spätmetastasen zu entnehmen. An dieser Stelle ist anzumerken, daß zwangsweise eine gewisse Übereinstimmung der Ergebnisse von Hüfner und Mitarb. mit den eigenen Resultaten bestehen muß, da es sich bei den von der Heidelberger Arbeitsgruppe untersuchten Fällen zum Teil um Würzburger Patienten handelte, die aus den eingangs erwähnten Gründen zur Durchführung der Radiojodtherapie in die Universitäts-Strahlenklinik Heidelberg überwiesen worden waren. Nach den Literaturangaben und den Erfahrungen aller mit der Nachsorge des Schilddrüsenkarzinoms Betrauten ist also davon auszugehen, daß es beim differenzierten Schilddrüsenkarzinom zum Teil auch nach sehr langen freien Intervallen noch zu einer Metastasierung kommen kann.

Den in Tab.7 aufgeschlüsselten Angaben verschiedener Arbeitsgruppen ist zu entnehmen, daß die Metastasen bei rund $20 \%$ der Fälle kein Radiojod anreichern. In der weiteren Literatur werden z. T. aber auch häufiger nicht radiojodspeichernde Metastasen beschrieben; die Häufigkeitsangaben bewegen sich hier zwischen 20 und $40 \%(14,20,24,27,32$, $36,37,51,59)$. Hüfner und Mitarb. $(22,23)$ finden bei ihren Patienten mit Primärmetastasen des differenzierten Schilddrüsenkarzinoms in $20 \%$ der Fälle keine Radiojodspeicherung; im eigenen Untersuchungsgut beträgt diese Häufigkeit rund $30 \%$.
Nach unserer Kenntnis stammt die einzige systematische Analyse des Jodspeicherverhaltens von Spätmetastasen des differenzierten Schilddrüsenkarzinoms von der Heidelberger Arbeitsgruppe. Nach den Angaben von Hüfner und Mitarb. $(22,23)$ liegt der Anteil der nicht radiojodspeichernden Spätmetastasen bei rund $2 / 3$; diese relative Häufigkeit deckt sich ziemlich exakt mit den eigenen Untersuchungsergebnissen. Es ist allgemein bekannt, daß insbesondere spät aufgetretene Metastasen eine geringere „funktionelle Differenzierung" aufweisen können als der Primärtumor $(18,20,32,36)$. Danach muß man davon ausgehen, daß der diagnostische Stellenwert der Radiojodszintigraphie zur Erfassung von Spätmetastasierungen im Vergleich zur Aussagekraft bei Primärmetastasen gering ist. Dies gilt auch für die nach unserer Auffassung optimale Untersuchungstechnik mit Frühszintigrammen $24 \mathrm{~h}$ und Spätszintigrammen $72 \mathrm{~h}$ nach Applikation der aufgrund der Strahlenschutzvorschriften ambulant maximal möglichen Dosis von $2 \mathrm{mCi}^{1.31} \mathrm{~J}$.

Radiojodszintigraphie oder Thyreoglobulin-RIA zur Verlaufskontrolle des differenzierten Schilddrüsenkarzinoms

Ganzkörperszintigramme mit ${ }^{131}$ Jod werden beim differenzierten Schilddrüsenkarzinom auch in Zukunft unerläßlich zur Bestätigung der Ablation des Schilddrüsenrestes bzw. von speichernden Primärmetastasen sein. Diese Feststellung wird durch den hohen Anteil von radiojodspeichernden Primärmetastasen und die Erfahrungen anderer Autoren $(23,24,49)$ gestützt. Der Stellenwert der ${ }^{131}$ Jod-Ganzkörperszintigraphie zur Erfassung von Spätmetastasen des differenzierten Schilddrüsenkarzinoms ist jedoch demgegenüber überraschend gering. Aus diesem Grunde sind bei Patienten mit differenziertem Schilddrüsenkarzinom, bei denen alles speichernde Schilddrüsengewebe durch die Operation und die Nachbehandlung mit Radiojod entfernt wurde, in der Spätphase der Nachsorge zur ${ }^{131}$ Jod-Szintigraphie alternative diagnostische Methoden verstärkt anzuwenden. $\mathrm{Zu}$ diesen diagnostischen Verfahren gehört in erster Linie die regelmäßige Bestimmung des Tumormarkers Thyreoglobulin im Serum.

Nach den bisherigen eigenen Untersuchungen $(6,40,41,42$, 44) und den Literaturangaben (vgl. Tab. 7) kann davon ausgegangen werden, daß die Sensitivität des Thyreoglobulin-RIA zur Erfassung von Metastasen knapp oberhalb von $90 \%$ liegt. Diese relativ hohe diagnostische Treffsicherheit gilt im Gegensatz zur Radiojodszintigraphie auch für Spätmetastasierungen (vgl. Abb. 4).

Von den 23 in Tab. 7 zitierten Arbeitsgruppen, die Empfehlungen zu einem Nachsorgeprogramm für das differenzierte Schilddrüsenkarzinom abgeben, schlagen 12 die bevorzugte Bestimmung des Tumormarkers Thyreoglobulin vor, während 10 Gruppen die Kombination der ${ }^{131}$ Jod-Ganzkörperszintigraphie mit der Thyreoglobulin-Bestimmung empfehlen. Weitere 4 Arbeitsgruppen entscheiden sich für die kombinierte Anwendung $(3,24,38,44)$. In einer neueren Publikation wird der noch näher zu prüfende Vorschlag gemacht, den Thyreoglobulin-RIA durch ${ }^{201}$ Thallium-Ganzkörperszintigramme zu ergänzen; auch wir konnten in Einzelfällen (insbesondere bei onkozytären Karzinomen) eine ${ }^{201}$ Thallium-Anreicherung in nicht radiojodspeichernden Metastasen feststellen.

Wir entscheiden uns heute für eine Kombination von Thyreoglobulin-Bestimmung mit ${ }^{131}$ Jod-Ganzkörperszintigramm (Abb. 5) in der Weise, daß in der Primärphase der Nachsorge ${ }^{131}$ Jod-Ganzkörperszintigramme so lange erforderlich sind, bis die vollständige Ablation allen speichernden Schilddrüsengewebes (sowohl von Restgewebe im Halsbereich als auch von 
Tab. 7 Serum-Thyreoglobulin ( $\mathrm{hTg}$ ) und ${ }^{131} \mathrm{~J}$-Ganzkörperszintigraphie beim differenzierten Schilddrüsenkarzinom. Übersicht über die Ergebnisse in der Literatur.

\begin{tabular}{|c|c|c|c|c|c|c|c|c|c|}
\hline \multirow{3}{*}{ Autor } & \multirow{3}{*}{ Jahr } & \multicolumn{6}{|c|}{ Ergebnisse bei Metastasen } & \multicolumn{2}{|l|}{ Empfehlungen } \\
\hline & & \multicolumn{2}{|c|}{$h T g-R \mid A$} & $\oplus$ & $\oplus$ & $\mathrm{hTg}_{\ominus}$ & $\Theta$ & \multirow[b]{2}{*}{$\begin{array}{l}\text { bevorzugte Nach- } \\
\text { sorgeuntersuchung }\end{array}$} & \multirow[b]{2}{*}{$\begin{array}{l}\text { Thyroxin } \\
\text { absetzen? }\end{array}$} \\
\hline & & Sensitivität & TSH-Abhängigk. & $\oplus$ & $\Theta$ & $\stackrel{131}{J}$ & $\Theta$ & & \\
\hline van Herle & $1975(57)$ & $6 / 6$ & - & - & - & - & - & - & -- \\
\hline Hagemann & $1977(19)$ & $28 / 28$ & - & - & - & - & - & - & - \\
\hline Denney & $1978(13)$ & $17 / 18$ & - & 17 & 1 & 0 & 0 & $h T g+131 \mathrm{~J}$ & - \\
\hline Okeriund & $1978(34)$ & $24 / 24$ & - & 22 & 2 & 0 & 0 & $\mathrm{hTg}+131 \mathrm{~J}$ & - \\
\hline Logerfo & $1979(29)$ & $23 / 23$ & - & 21 & 2 & 0 & 0 & - & - \\
\hline Shlosberg & $1979(54)$ & $15 / 16$ & - & - & - & - & - & - & - \\
\hline Tang Fui & $1979(56)$ & $17 / 17$ & - & 17 & 0 & 0 & 0 & $n \mathrm{Tg}$ & nein \\
\hline Böttger & $1980(8)$ & $30 / 34$ & + & 30 & 0 & 0 & 4 & $\mathrm{hTg}$ & nein \\
\hline Charles & $1980(11)$ & $8 / 8$ & + & - & - & - & - & $n T g$ & ja \\
\hline Eber & $1980(15)$ & $32 / 32$ & - & - & - & - & - & - & - \\
\hline Lamberg & $1980(26)$ & $18 / 23$ & - & - & - & - & - & $\mathrm{nTg}$ & - \\
\hline McDougall & $1980(32)$ & $3 / 3$ & $1 / 3$ & - & - & - & - & $n T g+131 \mathrm{~J}$ & ja \\
\hline Pacin! & $1980(35)$ & $45 / 47$ & + & 31 & 14 & 0 & 2 & $\mathrm{hTg}$ & nein \\
\hline Schlumberger & $1980(50)$ & $12 / 12$ & + & 8 & 4 & 0 & 0 & - & ja \\
\hline Weissel & $1980(58)$ & $10 / 10$ & - & 7 & 3 & 0 & 0 & $\mathrm{hTg}+131 \mathrm{~J}$ & - \\
\hline Ashcraft & $1981(1)$ & $19 / 19$ & + & 13 & 6 & 0 & 0 & $\mathrm{nTg}$ & nein \\
\hline Black & $1981(5)$ & $56 / 67$ & + & - & - & - & - & $\mathrm{hTg}$ & nein \\
\hline Colacchio & $1981(12)$ & $34 / 37$ & $5 / 37$ & 34 & 3 & 0 & 0 & $\mathrm{hTg}+131 \mathrm{~J}$ & ja \\
\hline Jänsch & 1981 (25) & $28 / 31$ & + & 26 & 2 & 3 & 0 & $\mathrm{nTg}$ & nein \\
\hline Schneider & $1981(51)$ & $22 / 22$ & $3 / 4$ & 18 & 4 & 0 & 0 & $h T g+131 \mathrm{~J}$ & ja \\
\hline Barsano & $1982(2)$ & $6 / 6$ & + & 4 & 2 & 0 & 0 & $\mathrm{hTg}$ & ja \\
\hline Botsch & $1982(9)$ & $23 / 24$ & - & 21 & 1 & 0 & 0 & $\mathrm{hTg}$ & - \\
\hline Castagnoli & $1982(10)$ & $13 / 23$ & $10 / 23^{*}$ & 9 & 3 & $10^{*}$ & 0 & $\mathrm{hTg}+131 \mathrm{~J}$ & ja \\
\hline Echenique & $1982(16)$ & $14 / 18$ & - & 11 & 3 & 4 & 0 & $\mathrm{hTg}+{ }^{131} \mathrm{~J}$ & ja \\
\hline Galligan & $1982(17)$ & $16 / 16$ & $1 / 16$ & 14 & 2 & 0 & 0 & $\mathrm{hTg}$ & ja \\
\hline Hüfner & $1982(21)$ & $42 / 51$ & - & 26 & $1 \overline{6}$ & 5 & 4 & $\mathrm{hTg}$ & - \\
\hline Schatz & $1982(48)$ & $10 / 13$ & $1 / 13$ & 9 & 1 & 3 & 0 & $\mathrm{hTg}+131 \mathrm{~J}$ & ja \\
\hline Schreyer & $1982(52)$ & $12 / 15$ & - & 9 & 3 & 1 & 2 & $h T g+{ }^{131} \mathrm{~J}$ & - \\
\hline Steinsträsser & $1982(55)$ & $17 / 19$ & - & 16 & 1 & 2 & 0 & - & - \\
\hline Müller-Brand & $1983(33)$ & $6 / 6$ & + & 1 & 5 & 0 & 0 & $\mathrm{hTg}+{ }^{201} \mathrm{Tl}$ & nein \\
\hline
\end{tabular}

* wegen extremer Häufigkeit bei Zusammenfassung nicht berücksichtig!.

Metastasen) bewiesen ist. Nachdem dieser Erfolg der primären Therapie jedoch dokumentiert ist, kann für die Spätphase der Nachsorge der Thyreoglobulin-RIA als Alternative zur Radiojodszintigraphie Verwendung finden. Hierbei sind allerdings neben dem Thyreoglobulin-RIA auch weitere Nachsorgeuntersuchungen, wie Röntgenaufnahmen des Thorax, Skelettszintigramme mit ${ }^{99 \mathrm{~m}}$ Tc-MDP und unspezifische Laboruntersuchungen (BSG, alkalische Phosphatase, Gamma-GT etc.), nicht zu vergessen. Zumindest beim onkozytären Karzinom kann auch die Ganzkörperszintigraphie mit ${ }^{201}$ Thallium von Nutzen sein. Findet sich bei einem Patienten nach vollständiger Ablation allen speichernden Schilddrüsengewebes ein Thyreoglobulinspiegel oberhalb der Nachweisgrenze des verwendeten Assays oder deuten die Ergebnisse der übrigen Nachsorgeuntersuchungen auf eine Metastasierung hin, so ist sofort eine Radiojodszintigraphie zur Fahndung nach evtl. speichernden Metastasen zu veranlassen.

Ein solches diagnostisches Programm wirft allerdings 2 Probleme auf:
1. An den verwendeten Thyreoglobulin-RIA sind höchste Ansprüche bezüglich der Nachweisempfindlichkeit und Reproduzierbarkeit zu stellen $(23,28,42)$.

2. Die Höhe des Thyreoglobulin-Serumspiegels ist abhängig vom TSH-Spiegel.

Alle Arbeitsgruppen, die sich mit der TSH-Abhängigkeit des Serum-Thyreoglobulins beschäftigt haben, finden eine entsprechende Korrelation (vgl. Tab.7); in einigen Fällen ist sogar nach den eigenen Ergebnissen und den Literaturmitteilungen mit einer vollständigen Suppression des Thyreoglobulins unter TSH-suppressiver Schilddrüsenhormontherapie zu rechnen. Aus diesem Grunde halten 10 von 17 Arbeitsgruppen, die dieser Frage nachgegangen sind (vgl. Tab. 7), Bestimmungen des Serum-Thyreoglobulins unter endogener TSH-Stimulation zur Ausschöpfung der optimalen Aussagekraft dieses Tumormarkers für erforderlich. In der weiteren Literatur schließen sich noch mindestens 5 Arbeitsgruppen dieser Ansicht an (3, $24,38,46,49)$.

Obwohl diese Forderung einen potentiellen Vorteil des Thy- 
reoglobulin-RIA im Vergleich zur ${ }^{131}$ Jod-Szintigraphie als Nachsorgeuntersuchung - nämlich die Vermeidung der für den Patienten häufig unangenehmen iatrogenen Hypothyreose ausschließt, glauben wir zur Zeit noch nicht auf regelmäßige Bestimmungen des Serum-Thyreoglobulins unter endogener TSH-Stimulation verzichten zu können. Die Zukunft muß zeigen, ob sich an größeren Patientenkollektiven der Eindruck der Heidelberger Arbeitsgruppe $(22,23)$ - den wir bisher teilen können - bestätigen läßt, daß die hTg-Bestimmung in Kombination mit den übrigen unspezifischen Nachsorgeuntersuchungen auch unter fortgesetzter Schilddrüsenhormonsubstitution ausreichend empfindlich zur Früherfassung von Spätmetastasen differenzierter Schilddrüsenkarzinome ist.

\section{Literatur}

(1) Ashcraft, M. W. A. J. van Herle: The comparative value of serum thy roglobulin measurements and ${ }^{131}$ Iodjne total body scans in the follow-up study of patients with treated thyroid cancer. Amer. J. Medicine 71 (1981) 806-814

(2) Barsano, Ch. P., C. Skosey, L. J. DeGroor, S. Refetoff: Serum thyroglobulin in the management of patients with thyroid cancer. Arch. Intern. Med. 142 (1982) 763-767

(3) Bednar, J., J. Nemec, V. Zamrazic, S. Vana, D. Pohunkova, S. Röhling: Serum thyroglobulin determinations in patients with differentiated thyroid carcinoma. Nucl. Med. 22 (1983) 204-211

(4) Beierwalters, W. H., R. H. Nishi yama, N. W. Thompson, J. E. Copp, A. Kubo: Survival time and "cure" in papillary and follicular thyroid carcinoma with distant metastases: Statistics following University of Michigan therapy. J. nucl. Med. 23 (1982) 561-568

(5) Black, E. G., A. Cassoni, T. M. D. Gimlette, C. L. Harmer, M. N. Maisey, G. D. Oates, R. Hoffenberg: Serum thyroglobulin in thyroid cancer. Lancet (1981) 443-445

(6) Börner, W., Chr. Reiners: Die Aufgaben des Nuklearmediziners in der Therapie des Schilddrüsen-Karzinoms. Med. Klin. 75 (1980) 80-87

(7) Börner, W., Chr. Reiners: Struma maligna: Behandlungsergebnisse und Nachsorge. Therapiewoche 32 (1982) 1049-1057

(8) Böttger, I., W. Dirr, W. Pabst: Erste Erfahrungen mit kommerziellen Thyreoglobulin(hTg)-RIA-Kits bei Struma maligna. NucCompact 11 (1980) 147-153

(9) Botsch, H.: Radioimmunoassay für die Serum-Thyreoglobulin-Bestimmung. Klinische Anwendung als Tumormarker bei der Verlaufskontrolle des differenzierten Schilddrüsenkarzinoms. Thieme, Sturtgart - New York 1982

(10) Castagnoli, A., G. Cappelli, A. D'Agata, S. Forni, S. Milani, A. Pupi: Can thyroglobulin assay really supplant radioiodine scans in patients with differentiated thyroid cancer? Lancet (1982) 573

(11) Charles, M. A., L. E. Dodson, N. Waldeck, F. Hofeldt, N. Ghaed, R. Telepak, J. Ownbey, P. H. Burstein: Serum thyroglobulin levels predict total body iodine scan findings in patients with treated well-differentia-
B. Kimmig: The significance of whole body scanning and plasma thyroglobulin measurements in the follow-up of merastatic thyroid cancer. Acta endocr. (Kbh.) Suppl. 246 (1982) 59-60 (2.3) Hüfner, M., H.-P. Stumpf, H. J. Hermann, B. Kimmig: Diagnostischer Wert des ${ }_{31}$ j-Ganzkörperszintigramms in der Nachsorge des differen zierten Schilddrüsenkarzinoms. Disch. med. W/schr. 108 (1983) 1234-1238

(24) Hundeshagen, H.: Post-operative diagnosis and therapy of thyroid carcinoma by nuclear medicine. Eur. J. nucl. Med. 8 (1983) 541-545

(25) Jänsch, A., H. G. Heinze, B. Hast: Serum-Thyreoglobulin ( $\mathrm{S}-\mathrm{hT} \mathrm{T}$ ). Ein Tumormarker bei Patienten mit differenziertem Schilddrüsenkarzinom. Strahlenther. 157 (1981) 381-392

(26) Lamberg, B.-A., T. Lahtinen, S. Dyrhönen, A. Kiuru, G. Wägar, M. Tikkanen, T. Mäkinen: Serum thy roglobulin, and radioiodine total body retention and scans in the detection of re-growth of differentiated thyroid carcinoma. In: Nuklearmedizin. Nuklearmedizin im interdisziplinären Bezug. Hrsg.: H. A. E. Schmidt, F. Wolf u. J. Mahlstedt. Schattauer, Sturtgart New York (1981) 971

(27) Leisner, B., W. Dirr, U. Büll, H. Langhammer, J. Lissner, H. W Pabst: Kombinationstherapie der Stru ma maligna, Ergebnisse 1960-1980. In: Neue Aspekte in Diagnostik und Therapie des Schilddrüsenkarzinoms Hrsg.: H. J. Biersack u. C. Winkler. Schattauer, Stuttgart - New York (1982) 161

(28) Lemish, I., F. Bennett, C. Martin, A. Warwick, M. Quinlan: A sensitive human thyroglobulin RIA to define clearly the presence or absence of functioning thyroid tissue. J. nucl. Med. 25 (1984) 49-55

(29) Lo Gerfo, P., R. Stillman, D. Co lacchio, C. Feind: Serum thyroglobulin and recurrent thyroid cancer. Lancet (1977) 881-882

(30) Mazzaferri, E. L., R. L. Young, Y. K. Maheshwari, C. Stratton Hill, Th. P. Haynie, R. C. Hickey, N. A. Samaan: ${ }^{131}$ I therapy in differentiated thyroid carcinoma: M. D. Anderson Hospital experience. Cancer 47 (1981) 664-671

(31) Mazzaferri, E. L., R. L. Young: Papillary thyroid carcinoma: A 10 year follow-up report of the impact of therapy in 576 patients. Amer. J. Med. 70 (1981) 511-518

(32) McDougall, I. R., M. F. Bayer: Follow-up of patients with differentiated thyroid cancer using serum thyroglobulin measured by an immunoradiometric assay. Comparison with ${ }^{131}$ ] total body scans. J. nucl. Med. 21

(1980) 741-744

(33) Müller-Brand, J., R. Fridrich, E. Spicher, J. J. Staub: Thyreoglobulin als Tumormarker und Thalliumszintigraphie zur Verlaufskontrolle bei differenzierten Schilddrüsenkarzinom. Schweiz. med. Wschr. 113 (1983) 325-327

(34) Okerlund, M. D., J. Sommers, B. Chuck, E. Lam, M. Galante, T. Hunt: Isotopic and serologic detection of thyroid cancer: ${ }^{131} 1$ scanning and serum thy roglobulin radioimmunoassay in 100 patients. J. nucl. Med. 19 (1978) 678

(35) Pacini, F., A. Pinchera, C. Giani, L. Grasso, L. Baschieri: Serum thyroglobulin concentrations and ${ }^{134}$ I whole body scans in the diagnosis of metasta- ses from differentiated thyroid carcinoma (after thyroidectomy). Clin. Endocrinol. (Oxf.) 13 (1980) 107-110

(36) Pochin, E.: Radioiodine therapy of thyroid cancer. Semin. nucl. Med. 1 (1971) 503-51s

(37) Pubi, H., A. Castagnoli, A. Mo rotri, G. La Cava, U. Meldolesi: Pro gnostic value of the ${ }^{131} \mid$ whole-body scan in postsurgical therapy for differentiated thyroid cancer. Cancer $\$ 2$ (1983) 439-441

(38) Referoff, S., E. C. Lever: The value of serum thyroglobulin measure ment in clinical practice. JAMA 250 (1983) 2352-2357

(39) Reimann, J.: Statistische Untersuchungen zur Prognose des Schilddrü senkarzinoms. Inaugural-Dissertation Würzburg (in Vorber.)

(40) Reiners, Chr.: Spezifische und unspezifische Tumormarker beim Schild drüsenkarzinom. Simultane Bestimmung von Thyreoglobulin, Kalzitonin, Carcinoembryonalem Antigen, AlphaFoetoprotein, Beta-Choriongonadotropin, Tissue Polypeptide Antigen, Immunglobulin E, Ferritin und Tennessee Antigen. TumorDiagnostik 2 (1981) 199-208

(41) Reiners, Chr.: Serum thyroglobulin as a substitution of ${ }^{131}$ I scans in follow-up of differentiated thyroid cancer? Acta endocr. (Kbh.) Suppl. 252 (1983) 66-67

(42) Reiners, Chr.: Serum-Thyreoglobulin und Thyreoglobulin-Antikörper. Ergebnisse beim Schilddrüsenkarzinom und anderen Schilddrüsenerkran kungen. Thieme, Stuttgart - New York 1983

(43) Reiners, Chr., W. Börner: Ein differenziertes Nachsorgeprogramm für das Schilddrüsenkarzinom. Med. Klin. 77 (1982) 34-41

(44) Reiners, Chr., W. Börner W. Wiedemann, W. Becker, F. Schick, W. Spegel, Chr. Eilles, W. Gerhards: Kritische Betrachtungen zur diagnostischen Wertigkeit der ThyreogiobulinBestimmung beim differenzierten Schilddrüsenkarzinom. In: Radioakti ve Isotope in Klinik und Forschung, Band 15/l. Hrsg.: R. Höfer u. H. Bergmann. Egermann, Wien (1982) 417 (45) Reiners, Chr., Th. Steurer, W. Börner, E. Moll: Erste Ergebnisse mit einem industriell hergestellten $\mathrm{Ra}$ dioimmunoassay für Thyreoglobulin: Untersuchungen an Patienten vor und nach Resektion blander Strumen sowie an Schilddrüsenkarzinom-Patienten. In: Schilddrüse 79. Hrsg.: D. Emrich, B. Glöbel u. B. Weinheimer. Thieme, Stuttgart - New York (1981) 111-119

(46) Roti, E., G, Robuschi, R. Ema nuele, P. Bandini, A. Russo, P. Riva, E. Galassi, U. P. Guerra, A. Manfredi, A. Bozzetti, A. M. Guazzi, A. Gnudi: The value of serum thyroglobulin measurement as a marker of cancer recurrence in the follow-up of patients previously treated for differentiated thyroid tumor. J. endocrinol. Invest. 5 (1982) 4.3-46

(47) Schäffer, R., Chr. Reiners, J. Reimann, W. Börner: Das onkozytäre Schilddrüsenkarzinom: Klinisch-pathologische Renaissance einer Tumorform? TumorDiagnostik 4 (1983) 161-168

(48) Schatz, H., S. Grebe, E. Mäser, J. Teuber, W. Horn, O. Schröder, Ch. Schatz: Serum-Thyreoglobulinspiegel als Tumormarker bei Schilddrüsenkarzinom. Klin. Wschr. 60 (1982) $457-464$ 
(49) Schatz, H., W. Horn, S. F. Grebe, H. Müller: Thyreoglobulinmessung oder ${ }^{13}$ Jod-Szintigraphie in der Nach sorge von Parienten mit differenzierrem Schilddrüsenkarzinom? Dtsch. med. Wschr. 45 (1983) 1737-1738 (50) Schlumberger, M., P. Charbord, P. Fragu, J. Lumbroso, C. Parmentier, M. Tubiana: Circulating thyroglobulin and thyroid hormones in patients with metastases of differentiated thyroid carcinoma: relationship to serum thyrotropin levels. J. clin. Endocr. 51 (1980) $513-518$

(51) Schneider, A. B., B. R. Line, I. M. Coldman, J. Robbins: Sequential serum rhyroglobulin determinations, ${ }^{131}$ | scans, and ${ }^{131}$ I uprakes after triodorhyronine withdrawal in patients with thyroid cancer. J. clin. Endocr. 53 (1981) 1199-1206

(52) Schreyer, T., D. Eissner

W. Grimm, K. Hahn: Serum-Thyreoglobulin (hT) zur Verlaufskourrolle bei Patienten mit Schilddrüsenkarzinom. In: Nuklearmedizin. Computergestützte funktionelle Analyse. Hrsg.: H. A. E. Schmidt u. H. Rösler. Schattauer, Stuttgart - New York (1982 839

(53) Schümichen, C., E. Schmitt: Be handlungsergebnisse in Freiburg. In: Neue Aspekre in Diagnostik und The rapie des Schilddrüsenkarzinoms Hrsg. H. J. Biersack u. C. Winkler. Schattaner, Sturtgart - New York (1982) 193

(54) Shlossberg, A. H., J. C. Jacobson, H. K. Ibbertson: Serum thyroglobulin in the diagnosis and management of thyroid carcinoma. Clin. Fndoer. (Oxf.) 10 (1979) 17-27

(55) Steinsträsser, A.: Erfahrungen mit der Thyreoglobulinbestimmung in Verlauf der Therapie und bei der Nachsorge von Patienten mit einem differenzierten Schilddrüsenkarzinom. ln: Neuc Aspekte in Diagnostik und
Therapie des Schilddrüsenkarzinoms. Hrsg.: H. J. Biersack u. C. Winkler. Schattauer, Stuttgart - New York (1982) 139

(56) Tang Fui, S., R. Hoffenberg M. N. Maisey, E. Black: Serum thyroglobulin concentration and whole bo dy radioiodine scan in follow-up of differentiated thyroid cancer after thyroid ablation. Brit. med. J. 2 (1979) 298-300

(57) Van Herle, A. J., R. P. Utler:

Elevated serum thyroglobulin. A mar ker of metascasis in differentiated thy

Priv.-Doz. Dr. Chr. Reiners Abteilung für Nuklearmedizin (Leiter: Prof. Dr. W. Börner) der Universität Würzburg

Josef-Schneider-Str. 2

D-8700 Wiirzburg roid carcinoma. J clin. Invest. 56 (1975) 272-277

(58) Weissel, M., H. Bergmann, R. Höfer: Klinische Wertigkeit von Serumthyreoglobulin- und ${ }^{131} \mathrm{~J}$-Ganz körperretentionsmessungen bei der Metastasensuche von differenzierten Schilddrüsenkarzinomen. Acta med. Austriaca 7 (1980) 114-119

(59) Young, R. L., E. L. Mazzaferri, A. J. Rahe, St. G. Dorfman: Pure follicular thyroid carcinoma: impact of therapy in 214 patients. I. nucl. Med. $21(1980) 733-737$ 